\title{
The Grounded Heightmap Tree. A New Data Structure for Terrain Representation
}

\author{
J. Alonso, R. Joan-Arinyo, L. Solano \\ Grup d'Informàtica a l'Enginyeria \\ Escola Tècnica Superior d'Enginyeria Industrial de Barcelona \\ Universitat Politècnica de Catalunya \\ Av. Diagonal 647, $8^{a}$, E-08028 Barcelona
}

Terrain modeling is a fast growing field with many applications such as computer graphics, resource management, Earth and environmental sciences, civil and military engineering, surveying and photogrammetry and games programming. One of the most widely used terrain model is the Digital Elevation Model (DEM). A DEM is a simple regularly spaced grid of elevation points that represent the continuous variation of relief over space. DEMs require simple storage and are compatible with satellite data. However, they do not easily account for overhangs.

In this work we report on the Grounded Heightmap Tree, a new data structure for terrain representation built as a generalization of the DEM. The new data structure allows to naturally represent terrain overhangs. We illustrate the performance of the Grounded Heightmap Tree when applied to represent terrains that undergo big changes.

\section{INTRODUCTION}

Terrain modeling is a fast growing field with many applications such as computer graphics, resource management, Earth and environmental sciences, civil and military engineering, surveying and photogrammetry and games programming. Since, in general, terrain models store huge amount of data, and applications must be highly responsive, having a model that allows quick answers to the queries on it would be paramount.

One of the terrain models most widely used is the Digital Elevation Model (DEM), also known as heightmap, that requires simple storage, is compatible with satellite data and allow good and simple surface analysis. On the other, they are slow to compute and are severely limited by the need of the uniform sampling and do not easily account for overhangs.

This work reports on the development of a new data structure, called Grounded Heightmap Tree. to model and deal with terrains that change at a high rate to capture, for example, big changes resulting from geotectonic events. It is a generalization of the heightmap model and accounts for hangovers. 

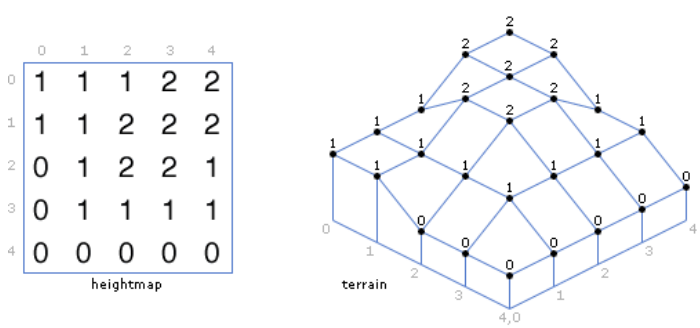

Figure 1: Digital Elevation Model. Left) Heightmap. Right) 3D terrain wireframe.

The manuscript is organized as follows. In Section 2 we briefly review the terrain models reported in the literature. In Section 3 we present the new terrain model along with the basic operations we have defined on it. The implementation and the preliminary results are presented in Section 4. We close with a brief summary in Section 5.

\section{TERRAIN MODELS}

Basically, terrain models belong to one one of three categories: Digital Elevation Models (DEM), Triangulated Irregular Networks (TIN) and Fractal Models. DEMs have been applied to represent terrains over uniformly distributed sample points. TINs allow representing real terrains with a high fidelity over irregular networks of sample points. Fractal Models are used to represent terrain models randomly generated.

\subsection{Digital Elevation Model}

DEMs are based on a simple structure named heightmap also known as heightfield. A heightmap is simply a 2D array of values which give the terrain elevations for ground positions sampled at regularly spaced horizontal intervals. Each value in the array represents the height of the terrain at that value's position. See Figure 1, [6]. For example, if the cell at $(2,3)$ has a value of 2 , then the terrain contains the point $(2,3,2)$.

On the one hand, DEMs require simple storage, are compatible with satellite data and allow good and simple surface analysis. On the other, they are slow to compute and are severely limited by the need of the uniform sampling and do not easily account for overhangs.

\subsection{Triangulated Irregular Networks}

A TIN is a DEM with a network of non-overlapping triangles whose vertices are placed at randomly located terrain points. These triangles are formed usually under Delaunay criterion. Irregular spaced sample points are measured with more points in areas of rough terrain and fewer in smooth terrain which results in an accurate representation of the terrain. Figure 2 shows a TIN, [4].

Pros of the TINS are that they need fewer points than DEMs for the same accuracy, their resolution naturally adapts to terrain roughness. Among the drawbacks we find that initial 


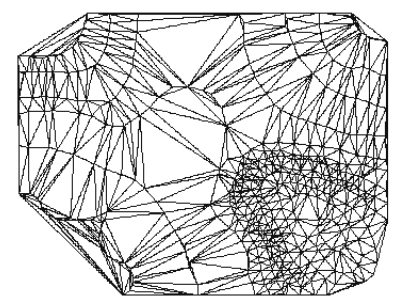

Figure 2: Triangulated Irregular Network.

construction is time consuming and some operations do not have efficient algorithms.

\subsection{Fractal Models}

Historically, fractals have been the second technique representation chosen by terrain rendering and visualization researchers. Examples of methods to generate fractal terrain models are diamond-square algorithm, fault algorithm and the hill algorithm. These techniques take as input a 2D array whose cells are properly initialized, and iteratively transforms it until a final terrain representation is reached.

\subsubsection{Diamond-square Technique}

The diamond-square algorithm is a method for generating highly realistic heightmaps for computer graphics, [5]. It is a three-dimensional implementation of the one-dimensional midpoint displacement algorithm which produces two-dimensional landscapes. Let us recall this algorithm.

The one-dimensional midpoint displacement algorithm starts with a single horizontal line segment. Then the midpoint of the line segment is computed and the old segment is replaced by the segments resulting from displacing in $\mathrm{Y}$ the midpoint by a random amount. Finally the range for possible random displacements is reduced. These steps are recursively applied to the line segments in the model until reaching the final model. Figure 3 illustrates the proces.

With this technique in mind, now we recall the diamond-square technique. We assume that the $2 \mathrm{D}$ array of points to hold the heigthmap is empty, square and with dimension $2^{n}+1$ with $n>1$. In what follows, a square is a set of four points as illustrated in Figure 4 Left, and a diamond is a set of four points as illustrated in Figure 4 Middle. For the diamonds we consider that the 2D array heightmap is circular as depicted in Figure 4 Right.

The technique is illustrated in Figure 5 on a simple example with a $5 \times 52 \mathrm{D}$ array. The

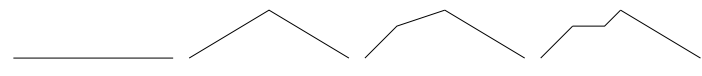

Figure 3: One-dimensional midpoint-displacement algorithm. 

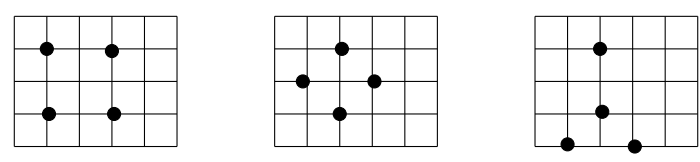

Figure 4: Diamond-square. Left: Square. Middle: Diamond. Rigth: Circular diamond.

starting point for the iterative technique sets the four corner points to the same height value. In Figure 5a the four corners are highlighted in black. Notice that what we have got is a square. Then there are two different possible steps: the diamond step or the square step.

The diamond step takes a square and generates a random value at the square midpoint, where the two diagonals meet. The midpoint value is calculated by averaging the four corner values, plus a random amount. This gives us diamonds when we have multiple squares arranged in a grid. See Figure 5b.

The square step takes a diamond and generates a random value at the center of the diamond. The midpoint value is calculated by averaging the corner values, plus a random amount generated in the same range as used for the diamond step. This gives us squares again. See Figure 5c. Since the array is circular, the midpoint corresponding to a diamond straddling over the array boundary gives value to two points on opposite sides of the array as illustrated in hollow points in Figure 6.

Now the algorithm iterates performing one diamond step, one square step and a reduction of the random values range until the length of the squares side is smaller than a given threshold. Figure 7 depicts the iterative process to generate de 3D terrain model.

\subsubsection{Fault Formation}

This technique is a very simple one, yet its results, although not the best, are pretty good, [3]. The technique is not limited to planar height fields, being also applicable to spheres to generate artificial planets. Hugo Elias [2], has posted tutorials on the application of this algorithm to spheres.

To start with we have a planar height field, where all points have zero height. Then we select a random line which divides the terrain in two parts (in general these parts will be different in size). The points to one side of the line will have their height displaced upwards, whereas the points on the other side will have their heights displaced downwards. Figure 8 illustrates the first iteration of the technique.

The cross points will have their height decreased, whereas the star points will have their

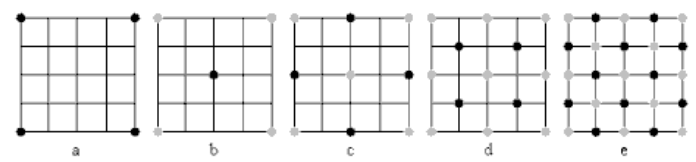

Figure 5: Diamond-square algorithm. 


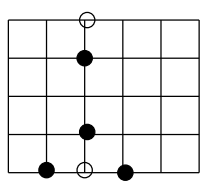

Figure 6: Diamond straddling over array boundaries.

height increased. So now we have a terrain with two distinct heights. If we keep dividing the terrain like this then we will get something that has valleys, mountains and so on. Figure 9 illustrates the process where the $2 \mathrm{D}$ array is recursively splitted.

\subsubsection{Hill Technique}

The hill technique can be seen as a kind of fault formation where the straight line used to divide the terrain is replaced with an sphere. The basic idea is simple, [6]. Start with a flat terrain (initialize all height values to zero). Pick a random point on or near the terrain, and a random radius between some predetermined minimum and maximum. Carefully choosing this min and max will make a terrain rough and rocky or smooth and rolling. Raise a hill on the terrain centered at the point, having the given radius. Repeat the two previous steps as many times as necessary. Notice that the number of iterations chosen will affect the appearance of the terrain.

Raising a hill is simple. A hill is kind of a rounded hump shape. The bigger the radius is, the taller the hill gets. Mathematically, it is a parabola revolved around the centerpoint, which reaches zero at the radius. Figure 10 depicts on the left an isolated hill and on the right the model after performing several iterations.

To generate a complete terrain, two things must be kept in mind. First, the equation shown above will yield a negative result if the point being calculated is farther from the centerpoint than the radius. When this happens, the negative value is ignored. Second, when two hills overlap their heights are added to each other. This gives nice amorphous lumps instead of obvious perfectly round bumps.

\section{THE GHT MODEL}

As presented in Section 2, heightmaps offer a good balance between complexity and performance. However, this balance is broken when trying to capture terrains with overhangs. The GHT model presented in this work is a generalization of the heightmaps that easily accounts

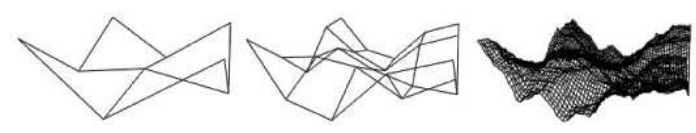

Figure 7: 3D terrain model generated with the diamond-square technique. 


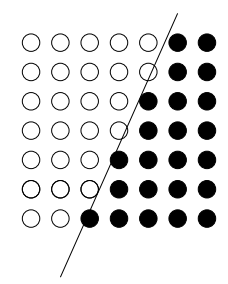

Figure 8: Fault formation. Dividing the height field.
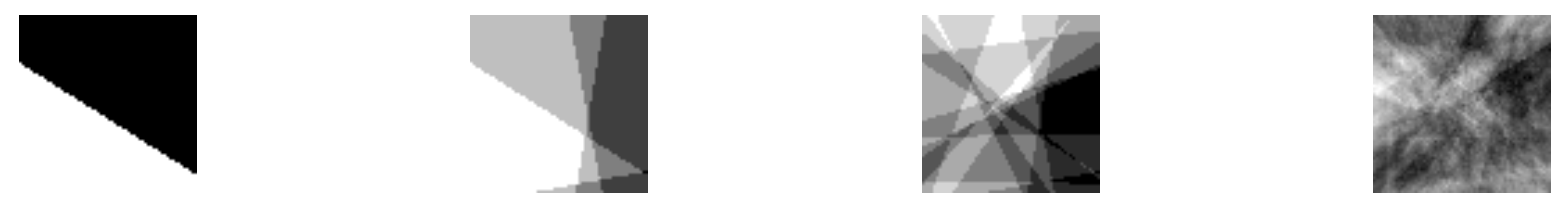

Figure 9: Fault formation. Terrain height map evolution as the number of iterations increases.
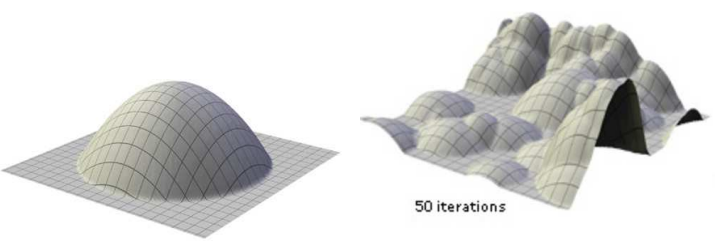

Figure 10: Left: Isolated hill. Right: The model after several iterations. 
for both overhangs and tunnels while basically keeping the simplicity of heightmaps. It is specially well suited to capture terrains built through a sculpting process from an initial $2 \mathrm{D}$ grid of terrain elevations.

\subsection{Definitions}

The model definition is rather simple. The grounded heightmap tree is a tree where the nodes are grounded heightmaps and the edges are pointers to other grounded heightmaps.

A grounded heightmap is a ground plane along with an axis alligned 2D array of data cells. The ground plane is defined by a point and the direction vector. Each data cell contains a height measured with respect to the ground plane along its direction vector, and a pointer to some data cell in a different grounded heightmap. To properly manage carvings and hangovers, the GHT model includes the type tag which distinguishes whether a grounded heightmap is an outdoor area, is an indoor area (carving or hangover) or is a heightmap which defines the transition between indoor and outdoor areas. Figure 11 shows the GHT datatype definition using C-like notation.

\subsection{Model Construction}

Constructing a GHT model starts with just one grounded heightmap whose ground plane is the $\mathrm{XZ}$ plane with the $\mathrm{Y}$ axis as direction vector, and whose $2 \mathrm{D}$ array of values gives the terrain elevations with respect the ground plane. No overhangs are included. The height data can be defined, for example, by hand or captured from satellite data. The links to grounded heightmaps are all null.

The initial GHT model is edited by applying a series of basic operations or events. The algorithm in Figure 12 illustrates this process. If ght is a properly initialized GHT model and $\mathrm{e}$ is the event to be applied, first the heightmap, hmap, on which the editing will take place is identified. hmap is the deepest heightmap in the GHT model such that includes the terrain region affected by the event and whose cells contain either a height or a valid link to a grounded heightmap. Then a set of new heightmaps configured according to the type of event, that we will define later on, is created. These heightmaps contain also information needed to place them with respect to the hmap heightmap. To apply the event means to assign to the new nodes the information that locally describes the terrain according to the event considered. Finally the new heightmaps are attached to the GHT model through the hmap.

Procedure ApplyEvent () considers two families of events: geotectonic and carvings. Geotectonic events include linear and radial erosions and, normal, inverse and lateral faults. Carvings include tunnels and caves. Each basic operation is defined by a set of parameters and the effect on the terrain will depend on the specific values assigned to them when the operation is triggered. Next we detail how each family of events is applied.

Figure 13 illustrates the geometry generated by the geotectonic events we consider. Linear erosion needs four grounded heightmaps and is defined by two points and an scalar. Points fix the position and length of the erosion, the scalar fixes the erosion depth. Radial erosion needs one grounded heightmap and is defined as an ellipsoid given by the radii and center. 


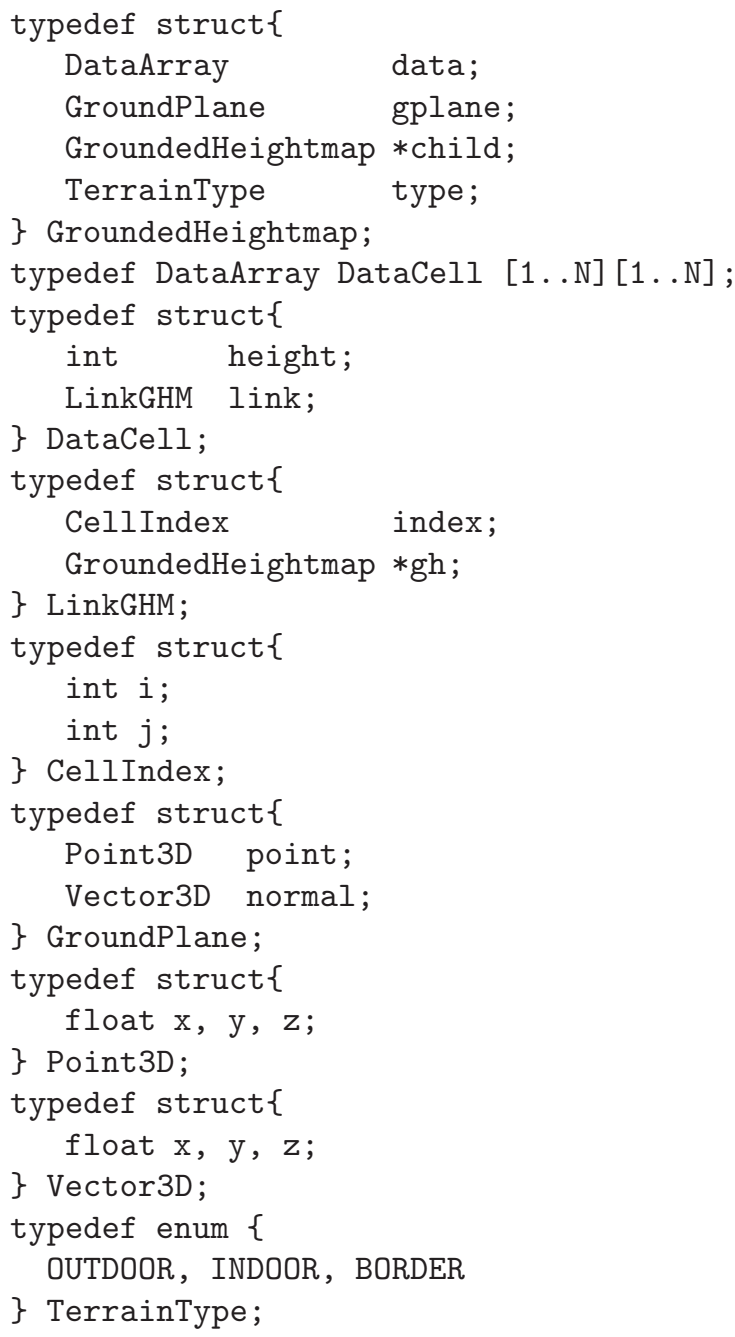

Figure 11: Grounded Heightmap datatype.

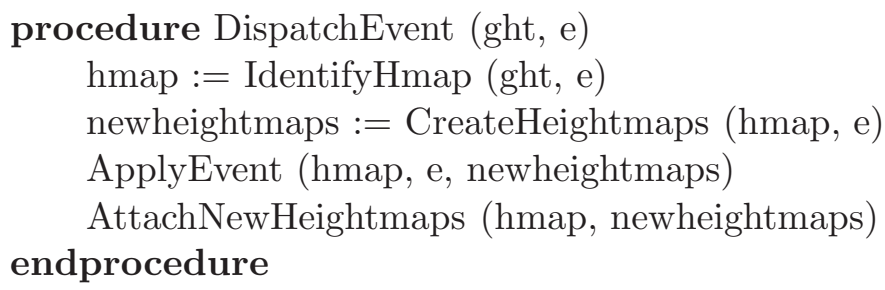

Figure 12: Editing the GHT. 

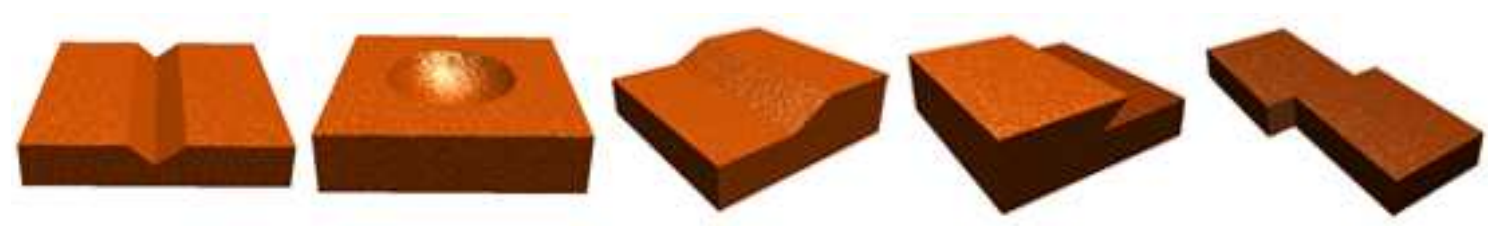

Figure 13: Geotectonic operations on GHT models.

Parameters in faults are defined by two points that define position and length, plus an scalar that fixes the terrain surface area affected by the event. Normal and inverse faults need three grounded heightmaps and lateral faults need two grounded heightmaps.

The algorithm in Figure 14 describes how geotectonic events are dealt with. First for each new heightmap in the event, the boundaries of the affected local terrain region are projected onto the XZ plane. Then for each cell in the 2D array of the new heightmap within the projected boundaries of the affected region, we apply the following process. If the current new heightmap and hmap have the same orientation and the hmap cell is a height, the new heightmap cell value is the height in hmap. If the hmap cell is a link, the new heightmap cell is a link to the considered hmap cell.

When the new heightmap and hmap have different orientations the situation is a little bit more complex. We have to assign to the cells in the new heightmap values taken from hmap. Since the groundplane of the new heightmap is at an angle with the hmap groundplane, in general, the $2 \mathrm{D}$ cell array of the new heightmap has more cells than that of hmap. Height values for the extra cells in the new heightmap are computed applying a simple linear interpolation.

After defining a new heightmap, those cells in hmap whose values have been transferred to the new heightmap no longer represent a valid height. Therefore the links in hmap point to the corresponding cells in the new heightmap. Finally to preserve GHT continuity each cell in the boundary of a new heightmap is linked to the neighbor cell in another heightmap.

Carving events are defined in two steps. First a protovolume is defined by sweeping a cross section along a rectilinear axis. Then the carving is generated by subtracting the protovolume from the terrain model.

The protovolume is characterized by two points, a polygonal cross section, and two scalars. The two points define both the axis and span of the protovolume. The polygonal cross section defines the carving cross shape. Each scalar is used to scale respectively the cross section to its actual size at the beginning and at the end of the protovolume. The number of grounded heightmaps in a carving tunnel is the number of sides in the polygonal cross section plus two heightmpas that define the indoor-outdoor borders. The groundplanes of these heightmaps are coincident with the XZ plane. In the carving cave, we have one heightmap that defines the cave entry and another one that defines the cave end. Carving events can be applied only on the GHT root node since so far we only support them on the XZ plane. Figure 15 left illustrates the shape generated by tunnel carving. Figure 15 right depicts the shape generated by carving a cave.

The carving process algorithm its outlined in Figure 16. First the algorithm extracts from the event heightmaps, newheightmaps, those that define the carving walls, iheightmaps, and 


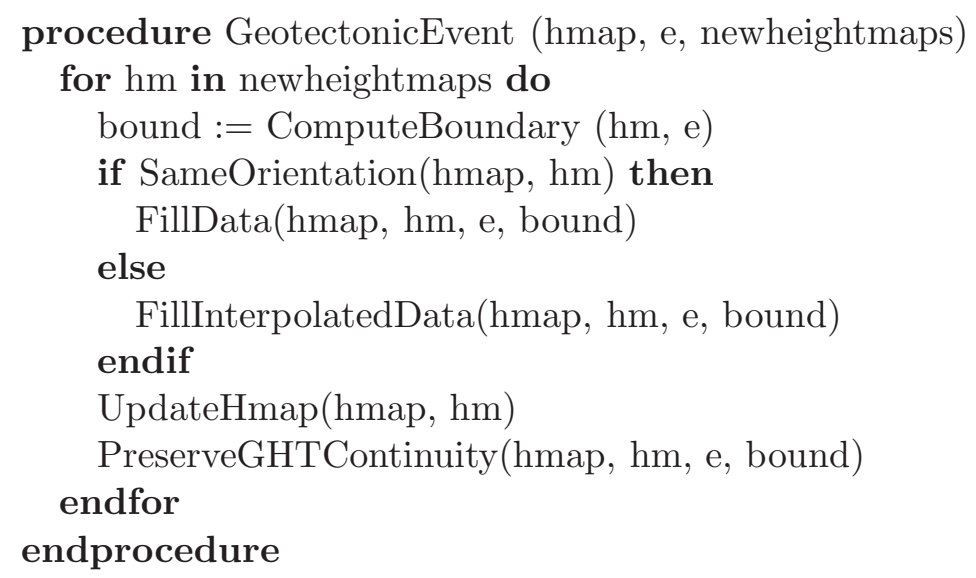

Figure 14: Geotectonic event algorithm.
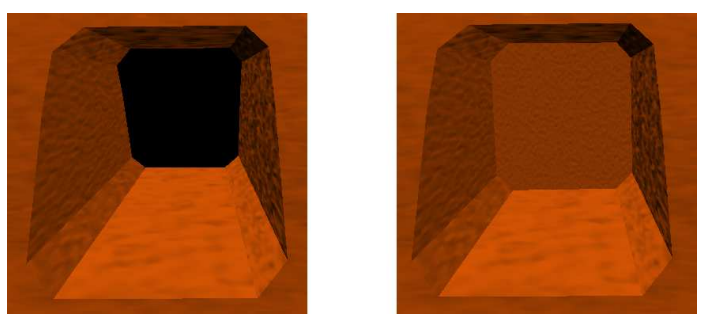

Figure 15: Carving events. 


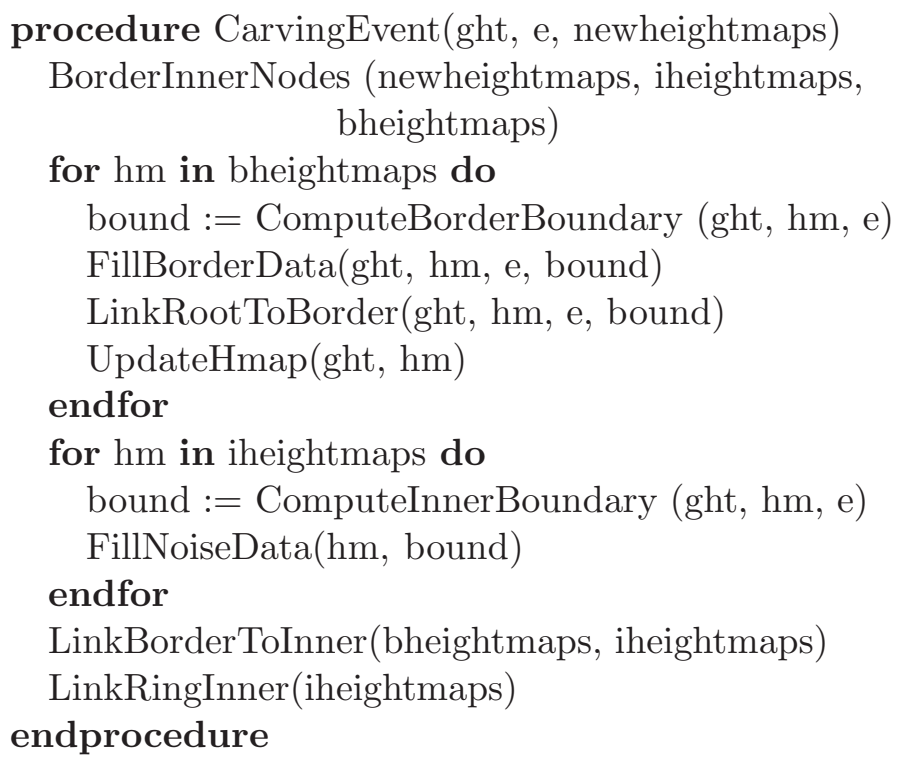

Figure 16: Carving process algorithm.

the heightmaps of the indoor-outdoor borders, bheightmaps.

For each indoor-outdoor border heightmap, first the set of cells that define the heights for the carving cross section, bound, is computed. To preserve model continuity, bound cells are linked to their neigbour cells in $\mathrm{hm}$. Heightmap cells where the cross section is projected are assigned as empty cells. After defining a border heightmap, those cells in hm corresponding to empty cells in the border heightmap must be labeled as null links.

For each wall heightmap first the set of cells that defines the height values is computed. Then the heights are defined as a smooth noise.

Finally, to preserve continuity, border-inner and inner-inner heighmaps links are stablished.

\section{IMPLEMENTATION AND RESULTS}

The algorithms have has been implemented on a Pentium M $1.73 \mathrm{GHz}$, with 1GB RAM, nVidia Geforce Go 6600 with 256 MB. The graphics API used was OpenGL and the GLUT library was used for events and window management. Digital elevation models consisted of heightfields with $128 \times 128$ cells.

To test the model performance, we have conducted two sets of experiments that we briefly describe in what follows. Related pictures and videos are available at [1].

\subsection{Single Events}

The first set of experiments consisted in applying a number of single events on a given terrain. Each event was applied on a fresh terrain. Figure 17 illustrates the results generated by linear 

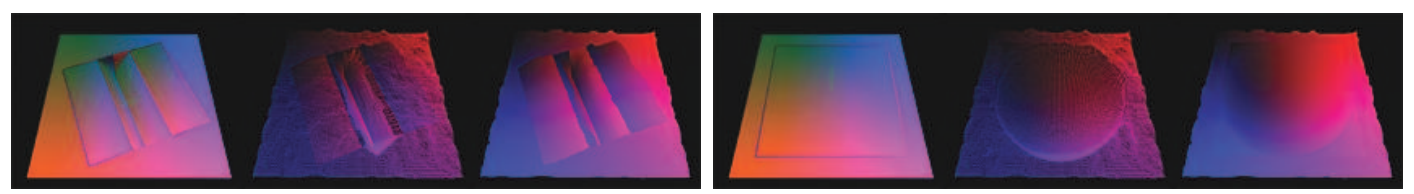

Figure 17: Linear and radial erosions. Left) Ground heightmap planes. Middle) 3D wireframe view. Right) 3D solid view.

and radial erosions and Figure 18 the results generated by the normal and inverse faults.

Figure 19 illustrates the results generated by carving a tunnel while Figure 20 shows the terrain after carving a cave.

Figure 21 shows the final terrain generated after applying two tunnel operations. On the left a $3 \mathrm{D}$ wireframe of the terrain is shown. On the right the terrain has been rendered applying textures on a skybox background.

\subsection{Sequence of Events}

The second set of experiments included a number of overlapping events applied in sequence to a given terrain yielding a complex model.

Pictures in the first row of Figure 22 show on the left side the fresh terrain as a wireframe and on the right side as a solid 3D view. In the middle row, Figure 22 shows the resulting terrain after applying a series of single editing operations. In the bottom, Figure 22 depicts the set of ground planes in the GHT model corresponding to the fresh terrain plus the series of operations.

The GHT tree structure corresponding to the terrain model shown in Figure 22 is given in Figure 23. Cells in gray are those that store the height according to the local ground plane. Cells in red are links to other GHT nodes. Blue cells are those cells whose data is not significative.

As a proof of concept, we have developed a small application to edit a fresh terrain by applying radial erosions. Each erosion is defined as the result of crashing a material particle on the terrain surface according to a fixed erosion law. Figure 24 shows the rain of material particles, depicted in yellow, crashing on the terrain represented from left to right by the ground planes, a wireframe and a textured terrain.
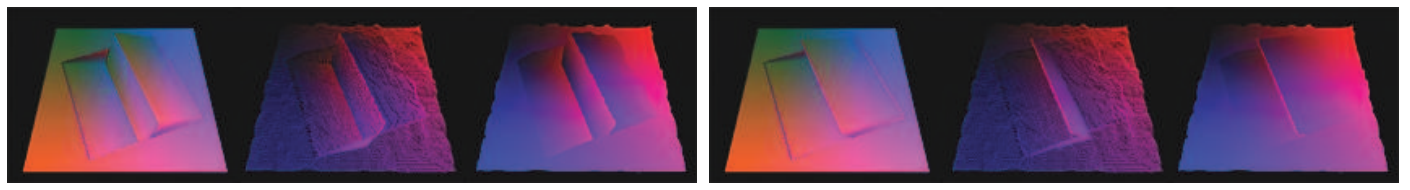

Figure 18: Normal and inverse faults. Left) Ground heightmap planes. Middle) 3D wireframe view. Right) 3D solid view. 


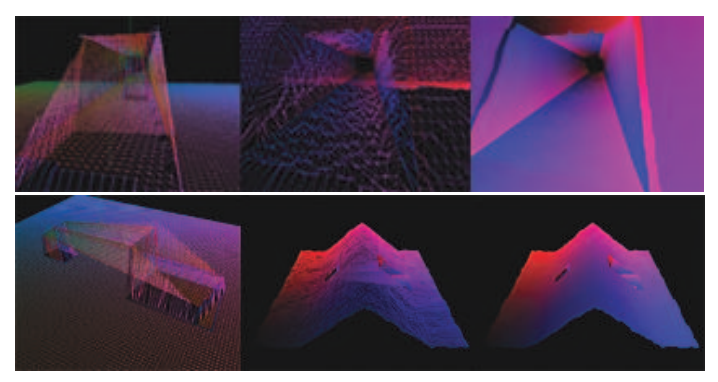

Figure 19: Carving tunnels. Left) Heightmap plane wireframe. Middle) 3D wireframe. Right) 3D solid view.

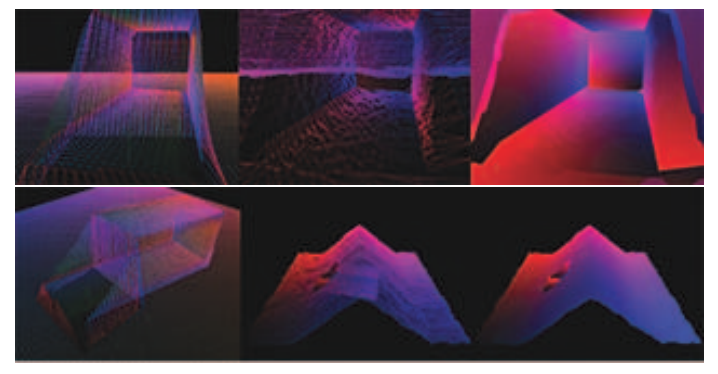

Figure 20: Carving caves. Left) Heightmap plane wireframe. Middle) 3D wireframe. Right) $3 \mathrm{D}$ solid view.
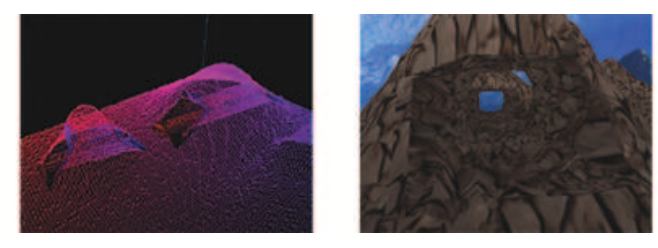

Figure 21: Terrain with two tunnels. Left) 3D wireframe view. Right) Textured 3D solid view with skybox. 

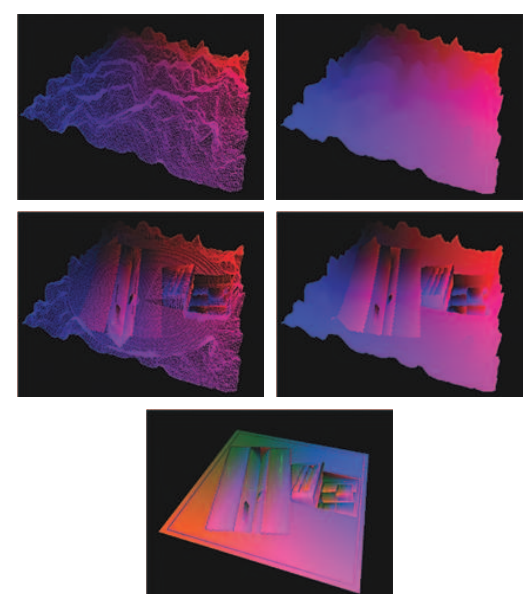

Figure 22: Complex model. Up left) Original heightmap 3D wireframe. Up right) Original heightmap 3D solid view. Middle left) Wireframe after applying some events. Middle right) Solid view after applying some events. Bottom) Set of ground planes in the final GHT.

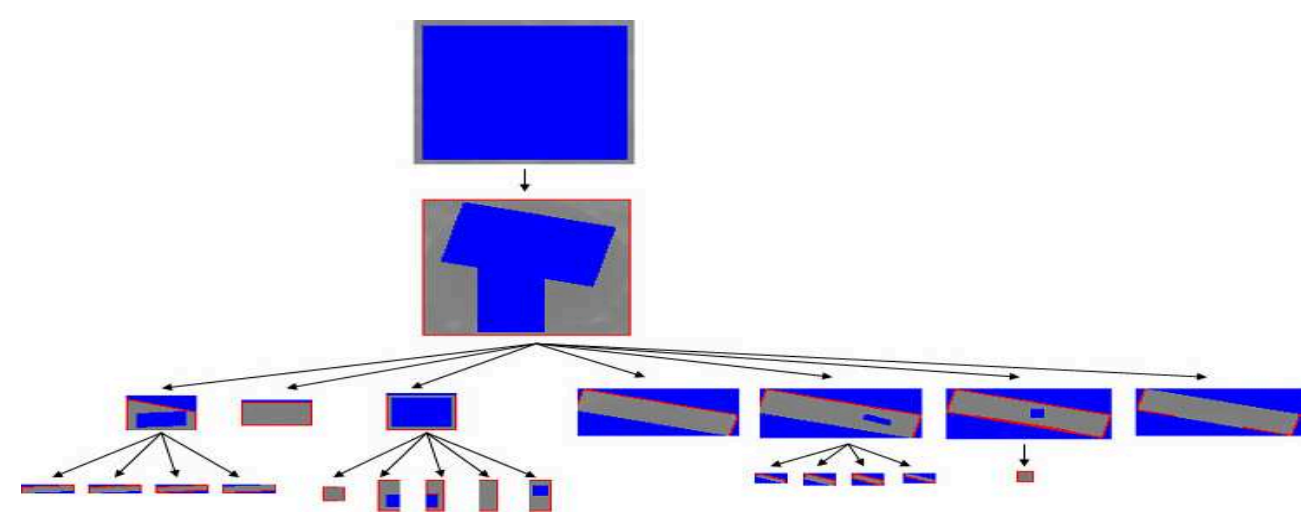

Figure 23: GHT tree of a complex model.

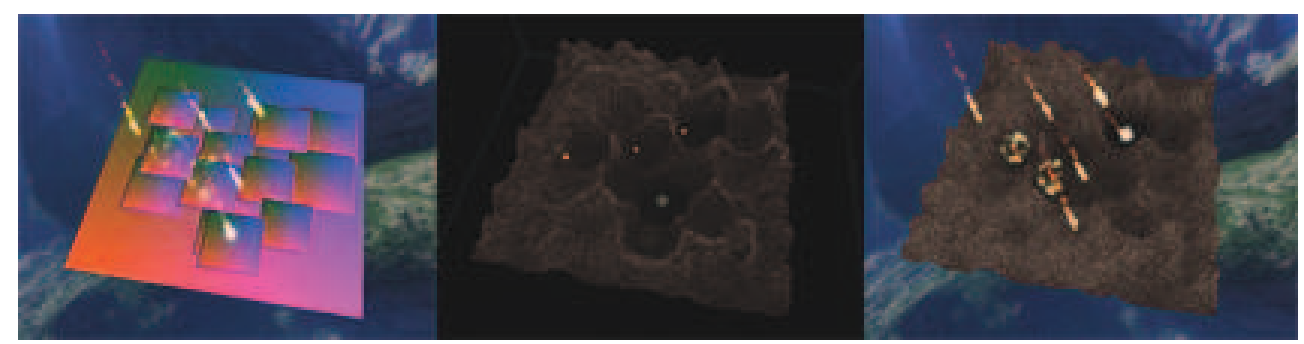

Figure 24: Landscape generated by shooting on a fresh terrain. 


\section{SUMMARY}

In this work we have proposed the Grounded Heightmaps Tree (GHT) as a new terrain model which is a generalization of heightmaps that overcomes limitations inherent to them like capturing terrain overhangs and carvings.

Besades, we have defined a set of operations to allow editing the terrain. There are two falimilies of operations: geotectonic events and carvings. The first family has been designed to mimic geotectonic events like linear and radial erosions; normal, inverse and lateral faults. The second family includes carving tunnels and caves.

We have conducted a series of experiments to test the model and associated operations performance. Preliminary results show that they are both effective and efficient.

\section{ACKNOWLEDGEMENTS}

This work has been partially funded by Ministerio de Educación y Ciencia and by FEDER under grant TIN2004-06326-C03-01.

\section{References}

[1] The Grounded Heightmap Tree. http://www.lsi.upc.edu/ jalonso/GHT.

[2] H. Elias. http://freespace.virgin.net/hugo.elias.

[3] The Fault algorithm. http://www.lighthoused3d.com/opengl/terrain.

[4] B. Klinkenberg. Digital elevation modeling. http://www.geog.buffalo.edu/arcinfo/aiwwwtut/step3.html.

[5] P. Martz. Generating random fractal terrain. http://www.gameprogrammer.com/fractal.html.

[6] Robot-frog. Terrain generation tutorial. http://www.robot-frog.com/3d.

\section{Bibliography}

Further reading on the subject.

1. J. Blow, Terrain rendering at high levels of detail, Game Developers Conference Proceedings, 2000.

2. P. Cignoni, F. Ganovelli, E. Gobbetti, Planet-sized batched dynamic adaptive meshes P-BDAM, IEEE Visualization'03, pp. 147-155, Roni Yagel and Gregory M. Nielson (Editors), IEEE Computer Society Press, 2003.

3. D. Cohen-Or, Y. Levanoni, Temporal continuity of levels of detail in Delaunay triangulated terrain, IEEE Visualization'96, pp. 37-42, R. Yagel and Gregory M. Nielson, IEEE Computer Society Press, 1996. 
4. W. De Boer, Fast terrain rendering using geometrical mipmapping, http://www.flipcode.com/articles/article geomipmaps.pdf, 2000.

5. M. Duchaineau, M. Wolinsky, D. Sigeti, M. Miller, C. Aldrich, D. Mineev-Weinstein, ROAMing terrain: Real-time optimally adapting meshes, IEEE Visualization'97, pp. 81-88, IEEE Computer Society Press, 1997.

6. M. Duchaineau, ROAM algorithm version 2.0, http://www. cognigraph.com/ROAM homepage/ROAM2/

7. H. Hakl, L. Van Zijl, Diamond terrain algorithm: Continuous levels of detail for height fields, South African Computer Journal, 2002, Vol. 29, pp. 81-88.

8. D. Hill, An efficient, hardware-accelerated, level-of-detail rendering technique for large terrains, PhD Thesis, University of Toronto, 2002.

9. H. Hoppe, Smooth view-dependent level-of-detail control and its application to terrain rendering, IEEE Visualization'98, pp. 35-42, IEEE Computer Society Press, 1998

10. J. Levenberg, Fast view-dependent level-of-detail rendering using cached geometry, IEEE Visualization'02, pp. 119-118, IEEE Computer Society Press, 2002.

11. P. Lindstrom, D. Koller, W. Ribarsky, L. Hodges, N. Faust, G. Turner. Real-time, continuous level of detail rendering of height fields, 23rd Annual Conference on Computer Graphics and Interactive Techniques, pp. 109-118, SIGGRAPH, 1996.

12. F. Losasso, H. Hoppe, Geometry clipmaps: Terrain rendering using nested regular grids, ACM Transactions on Graphics, Vol. 23, No. 3, pp. 779-776, 2004.

13. M. Pharr (Editor), GPU Gems 2, Addison-Wesley, 2005.

14. T. Polack, Focus on 3D Terrain Programming, A. LaMothe (editor), Premier Press, Game Development, Cincinnati, Ohio, 2003.

15. A. Pomeraz, ROAM using surface triangle clusters (RUSTiC), PhD dissertation, University of California at Davis, 1998.

16. S. Röttger, W. Heidrich, P. Slusallek, H.P. Seidel, Real-time generation of continuous levels of detail for height fields, 6th International Conference in Central Europe on Computer Graphics and Visualization, pp. 315-322, 1997.

17. C. Tanner, The clipmap: a virtual mipmap, 25th annual conference on Computer Graphics and interactive techniques, pp. 151-158, 1998.

18. T. Ulrich, Rendering massive terrains using chunked level of detail control. Draft, Course Notes, SIGGRPAH 2002.

19. D. Wagner, ShaderX2: Shader Programming Tips \& Tricks with DirectX 9, Wordware Publishing Inc., 2003.

20. Beautiful Landscapes By Means Of Height Mapping, http://nehe.gamedev.net/data/lessons/lesson.asp?lesson 
21. Virtual Terrain Project http://www.vterrain.org/

22. Efficient rendering of geometric data using OpenGL VBOs in SPECviewperf, http://www.spec.org/gpc/opc.static/vbo_whitepaper.html 